\title{
technology feature
}

\section{To wild depths for new models}

There are animals out there with unique phenotypes that could yield insight into human health and disease. Meet three such fishes from waters around the world: Antarctic icefish, Mexican cavefish, and Atlantic killfish.

\section{Jim Kling}

$\mathrm{n}$ the course of developing an animal model for osteoporosis, William

Detrich shattered his left hip. In 2018, the professor of biochemistry and marine biology at Northeastern University was far from a lab. He was aboard a research vessel in the Drake Passage, a frigid body of water that stretches between the southern tip of South America and the northern tip of Antarctica, when a violent wave rocked the ship and threw him off his top bunk. Instead of spending the field season at Palmer Station in Antarctica, he had to be Medevac'ed to Argentina and then back to Boston, where surgery and months of recovery awaited.

Why make this dangerous journey? For icefish. Icefish are unique animals, with porous bones that allow them to float in the water column to feed. That attribute, one of several unique features, makes them a unique kind of animal model. They display a phenotype without ill effect that, in humans, would be pathological. The remodeling that has occurred in icefish bones over millions of years, possibly including changes to regulatory elements that determine bone density and mineralization, may be similar to the mechanisms underlying osteopenia and osteoporosis over a human lifetime, says Detrich.

\section{"The fact is, we have model \\ systems for reasons," \\ says William Detrich. "But maybe they don't display the phenotype that you're really interested in. That's why you have to look beyond conventional models to what I sometimes call non-model model systems."}

Icefish aren't alone; as researchers look outside the lab, it's not hard to find other examples of animals with unique phenotypes. For example, Mexican river fish, cousins to the common aquarium Tetra, that moved into darkened, barren caverns

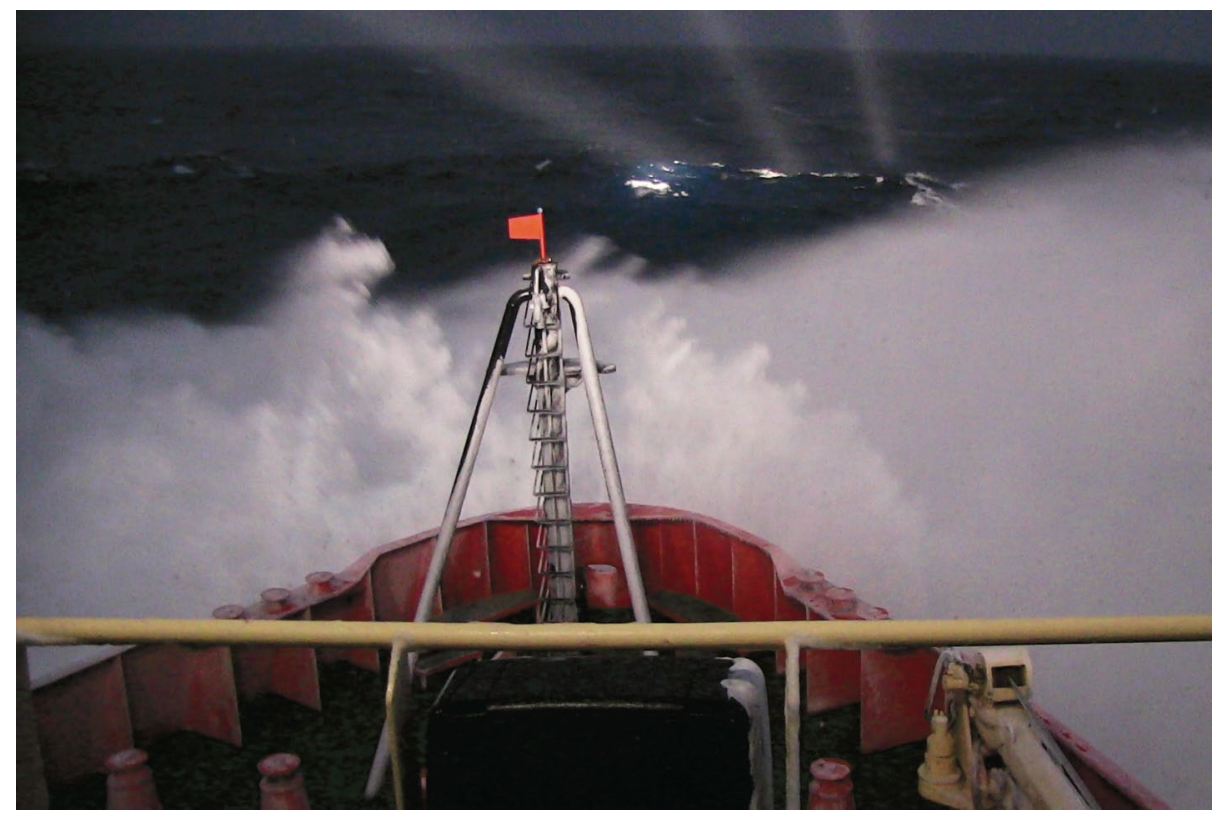

Worth the trip? The Antarctic Research and Supply Vessel Laurence M. Gould experiences stormy seas. The vessel has ferried William Detrich and others south to sample for icefish. Credit: H. William Detrich

have since evolved mechanisms to maximize nutrient use that evoke both human diabetes and risk factors for colon cancer; minnows living in waters off the northeastern US have managed to shrug off industrial toxins that could otherwise kill the developing embryo-a talent that could shed light on how smoking leads to lung cancer.

Such fishes are being examined as alternatives to the mice, rats, and other 'model' species that tend to dominate animal research. These familiar workhorses are popular, but results can disappoint. Deliberate genetic alterations to model a disease in a mouse or rat don't always account for the array of other changes that may accompany that disease in patients. They are artificial constructs that only imperfectly mimic human disease. As a result, positive results in these models often disappear when tested in humans.

Detrich and other researchers argue a need to take a fresh view. The ability to analyze the identity and expression of massive numbers of genes, proteins, metabolites, and other factors in model systems has granted scientists a much clearer understanding of genes and their networks. These days, genes can also be edited in almost any species. This is leading biology into a new era, according to Misty Riddle, a postdoctoral fellow in genetics at Harvard Medical School who works with Mexican cavefish.

Detrich certainly agrees. "The fact is, we have model systems for reasons, because they're usually manipulable in a genetic

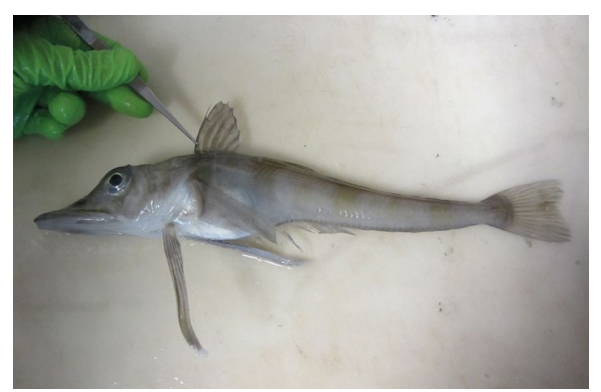

An icy catch: An Antarctic icefish. Credit: J. Postlethwait 


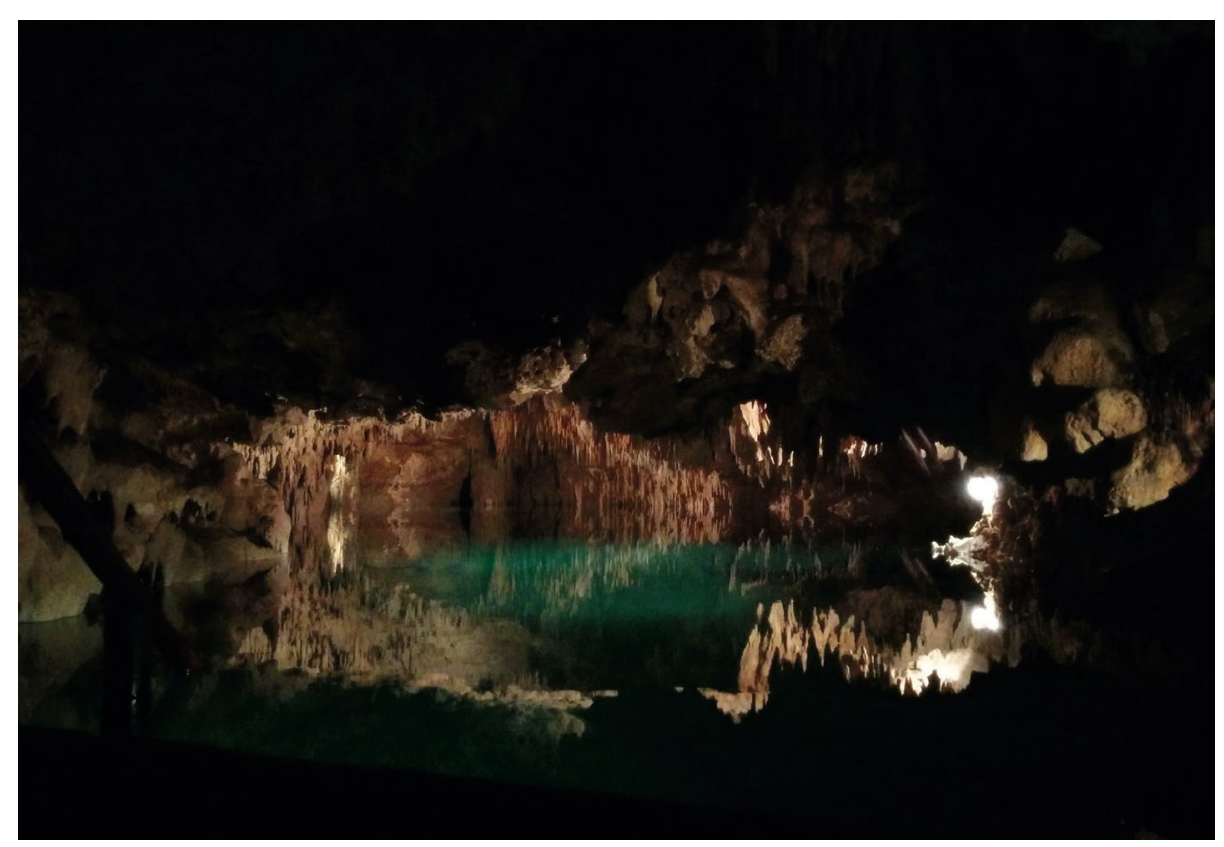

Into the dark: Mexican cavefish diverged from their river-remaining brethren in underwater rivers. Credit: Christian Escalante Castillo/EyeEm/Getty

sense, with all of the molecular tools that you can bring to bear. But maybe they don't display the phenotype that you're really interested in. That's why you have to look beyond conventional models to what I sometimes call non-model model systems."

\section{Out of unfamiliar territory}

Detrich has spent decades studying icefish, which evolved millions of years ago from ancestors that lived on the sea bottom. When he began working in the Antarctic in 1981, he initially studied structural microtubule proteins in the cells that manage to function in the frigid Antarctic waters, where temperatures can drop to $-1.9^{\circ} \mathrm{C}$, the freezing point of sea water. But soon another curiosity drew his attention. All 16 species of icefish, members of Channichthyidae

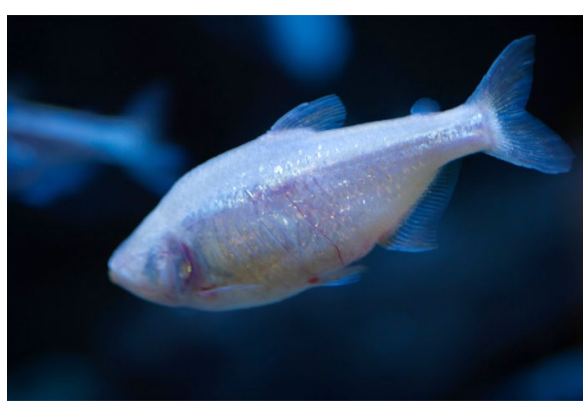

Uniquely adapted: An Mexican cavefish. Credit: Getty Images/iStockphoto family, lack hemogloblin. As a result, their blood is clear and opalescent, and they are alone among all vertebrates to bear this strange, anemic phenotype.

Astyanax mexicanus cavefish evolved more recently than icefish, but in another austere environment: stygian Mexican caves almost entirely devoid of nutrients. Sometime in the past 200,000 years, and probably on at least two separate occasions ${ }^{1}$, populations of Mexican tetra fish found themselves isolated in these dark chasms. There, they evolved a unique phenotype: they became blind and, in human terms, diabetic.

But they also "store fat like crazy," says Riddle. And they're insulin-resistant, leading to dramatic fluctuations in glucose levels during feeding and fasting. That's a problem in humans, but the fish don't seem troubled by it. Considering that nutrients only flow into the caves with seasonal flooding, it makes sense that they should gorge themselves.

Less exotic, but no less interesting to the researchers that study them, are Atlantic killifish, Fundulus heteroclitus. The species is extremely common in coastal rivers in the northeast U.S., but they live parochial existences: an individual fish likely travels no more than a few hundred yards in its lifetime, leading to many genetically distinct local populations.

Some live in rivers highly polluted from industrial and naval operations, but they show surprising resilience, according to Bryan Clark, an ORISE Research Fellow at the Oak Ridge Institute for Science and Education who also works for the Environmental Protection Agency. Specifically, killifish embryos develop normally despite high levels of polychlorinated biphenyls (PCBs) and polycyclic aromatic hydrocarbons (PAHs), both of which can lead to acute toxicity and cardiac teratogenicity in most vertebrates.

Like the icefish and the cavefish, the minnow-like killifish is a potential benefactor to human medical research, but it is also a resilient victim of humanity's excess. In each species, understanding its unique adaptations to its extreme environment could hold clues to understanding pathogies in people.

\section{What's in the genes?}

When global climage change and glaciation cooled the oceans, changes in bone structure allowed icefish to rise from the sea bottom into the water column to feed. The bones of the icefish resemble what happens in humans as they age, with decreasing skeletal mineralization that reduces bone density. But the fact that those changes occur in a human lifetime suggests that the culprit in people is not a change to a protein-coding gene but rather modifications to regulatory elements that control gene expression,

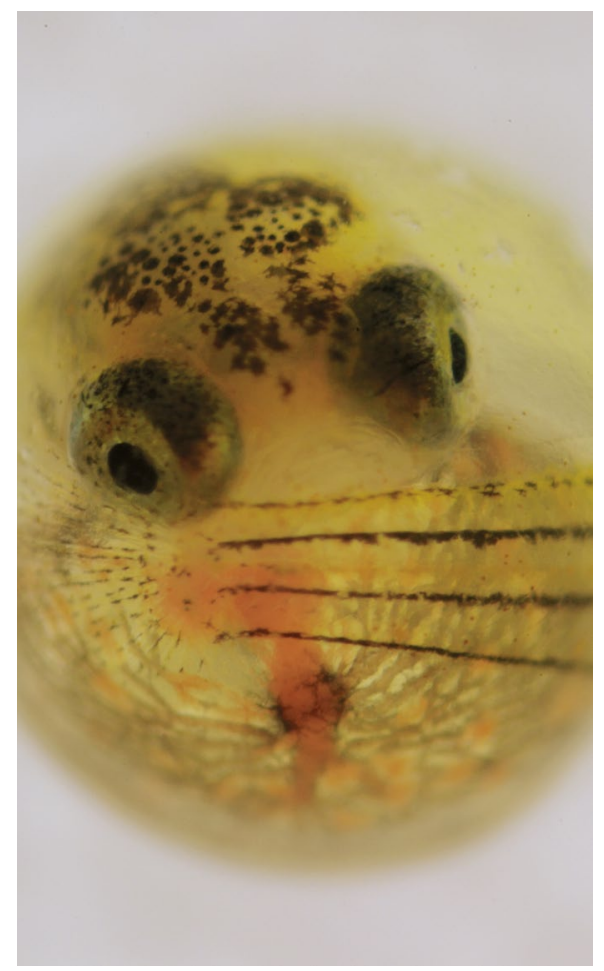

Pollution? No problem: An Atlantic killifish at 10 days post-fertilization. Credit: B. Clark 
causing either a decrease in expression of proteins that promote bone mineral density, or dialing up proteins that reduce it. If researchers can figure out how the icefish did it, the lessons might translate to treating bone density diseases of old age.

"It's a matter of comparing what happens in a person over developmental time to what's happening in a fish over evolutionary time," says John Postlethwait, a professor of biology at the University of Oregon.

But the icefish may have a tale to tell about more than just bone disease. The evolutionary changes that led to their striking blood color could lend insight into human anemia.

To investigate the connection, the researchers compared icefish to close relatives. Icefish are one of eight families in the notothenioid suborder; the other seven are red-blooded.

When the team looked at blood cell gene expression in icefish, they found that 15 of 16 icefish species had deletions in their alpha-globin genes; in the $16^{\text {th }}$ species "it was really messed up," says Detrich. His team then compared the transcriptome of blood cell progenitors from icefish and from their red-blooded relatives. That led to the discovery of genes that only the latter expressed, implying a role in the formation of red blood cells. Among those was a gene encoding a protein called hemogen that turns out to have critical-and wideranging-role in red blood cell formation; other researchers have discovered it in mice, rats, and humans, too ${ }^{2}$.

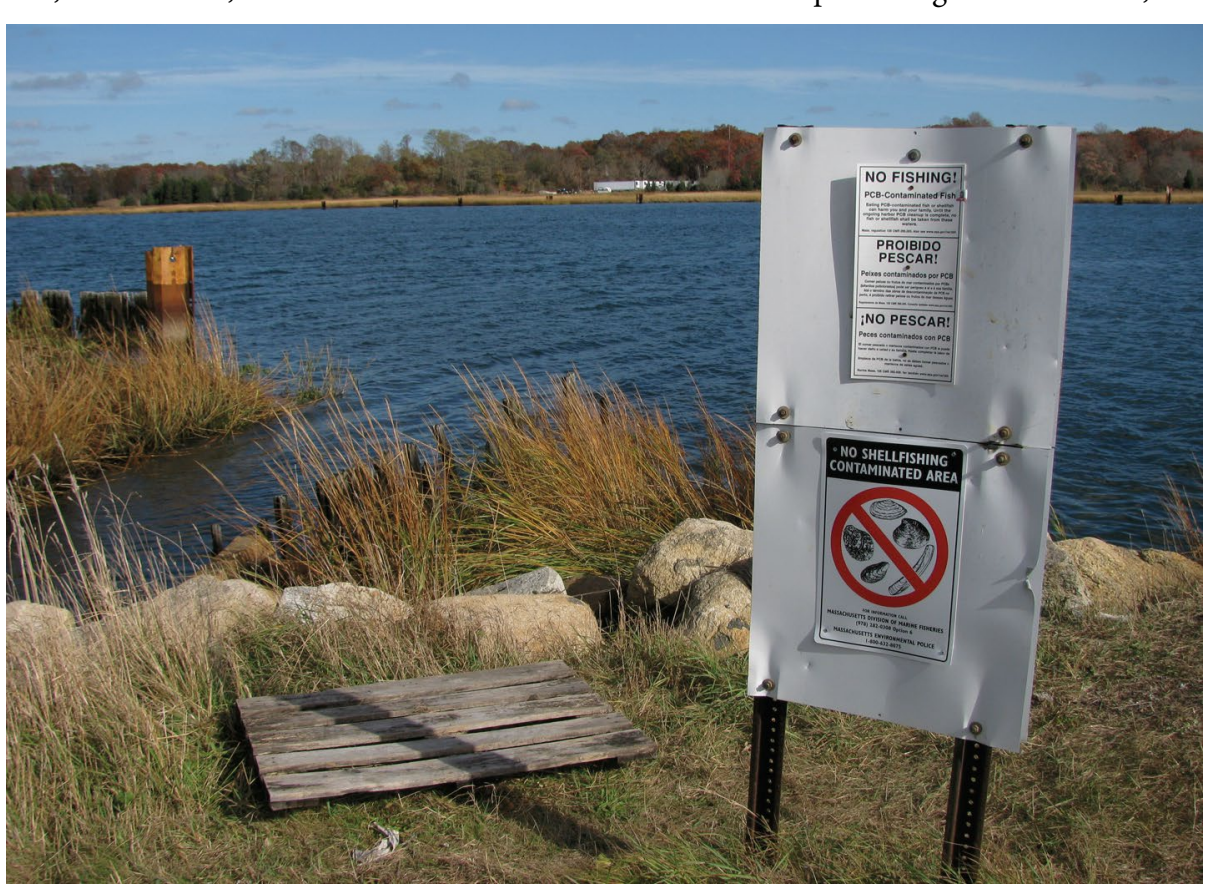

A familiar scene? A polluted waterway in New England. Despite the toxins, killifish embryos seem to develop just fine. Credit: B. Clark

\section{Box 1 | Back in the lab}

There are more than 30 known population of cavefish in Mexico's Sierra de El Abra region, according to Riddle. Collecting from some populations involves little more than a 5-minute hike and dipping a net into a cool, dark pool, but others can require repelling down entrance shafts and, in some cases, supplemental oxygen. Once back in the lab, the cavefish are easy to raise and care for. Most of Riddle's fish come from expeditions conducted over a decade ago. Inducing them to spawn is as simple as raising the temperature of their tanks a few degrees above the normal room temperature.

Collecting killifish is much simpler than sailing dangerous waters or repelling into darkened caverns. Researchers need only head to a marsh and take along a fisherman's bait trap-filled with frozen squid if they're feeling extravagant, but in bygone days the preferred lure was hot dogs. Clark's aquaculture setup includes flowing sea water to mimic tidal changes,

The next step is to compare whole genomes, a task that received a boost in February with the publication of the genome of the Antarctic Blackfin Icefish, Chaenocephalus aceratus 3 . "Now we can do comparative genomics to ask why it is that icefish are not producing red blood cells, them have also been making connections to
human conditions.

\section{Making human connections}

In 2018, Riddle and her colleagues published a paper showing that the cavefish has a mutation in the insulin receptor gene that reduces insulin binding. A similar change in humans leads to low weight and premature death $^{6}$. The team has gone on to investigate the cave fish's digestive system and how it has evolved to extract the maximum amount of nutrition from exceedingly rare meals.

The work could lead to insights into colorectal cancer. When raised in lab aquariums (Box 1), the researchers found that the cavefish have a longer gut than the river fish and much greater proliferation of cells in the intestinal epithelium, where nutrients get absorbed. "That was surprising to me because I see that process as energetically expensive, but under a fed condition, it's probably beneficial to have more surface area for nutrient absorption," says Riddle. When the fish is starved, those surfaces shrink. "They may be able to grow 


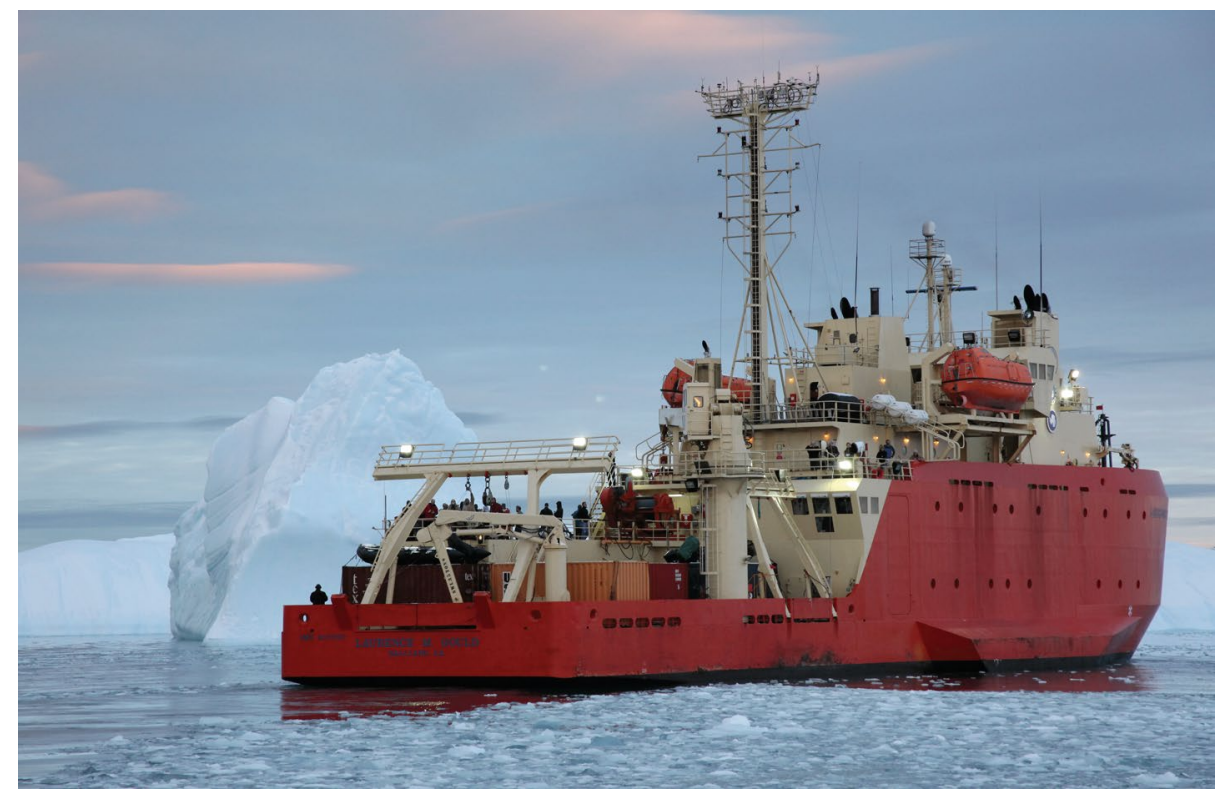

Sailing on: The Gould in calmer conditions. Credit: H. William Detrich

the gut when they need to and shrink it down when food isn't available. It seems that surface fish can't do this to the same extent," says Riddle.

Inter-breeding experiments with the river fish revealed a potential candidate gene, Kruppel-like factor 5 (KLF5), which is a known oncogene contribing to human gastrointestinal (GI) cancer. The team is now examining the muations found in human GI cancers to find out if similar mutations might occur in the cavefish. "In humans, there is interest in how the gut responds to different diets, since some diets may increase the chance of developing gastrointestinal cancers. That's an exciting link-how the cavefish responds to nutrients, and it seems to be really different from the river fish. We hope to relate that to humans and how certain diets may lead to overproliferation of the intestinal epithelium and potentially GI cancer," says Riddle.

The killifish may also shed light on cancer. Clark and his colleagues used genetic knockouts to determine that the aryl hydrocarbon receptor (AHR)-2 may play a central role in embryonic cardiac teratogenicity induced by a PCB and some PAHs. Once bound to a PAH or PCB, AHR-2 triggers the activation of drugmetabolism pathways, among other genes. When they knocked out AHR-2 in a toxin-sensitive killifish, the developing embryos were protected from the effects of the toxins ${ }^{7}$.
In work done as part of the Boston University Superfund program, a group led by Andrew Whitehead at the University of California San Diego and including Mark Hahn at the Woods Hole Oceanographic Institution compared hundreds of genome sequences from PCB-sensitive and PCBresistant killifish from different locations. They found evidence of evolutionary pressure on the AHR-2 gene and a binding partner, called aryl hydrocarbon receptorinteracting protein (AIP). Hahn believes that AIP helps to stabilize the AHR protein. Humans have AIP as well, and just one AHR. "AHR has been studied in humans as a possible susceptibility gene for cancer from smoking, or for birth defects from exposure to aromatic hydrocarbons, because it's involved in regulation of the drugmetabolizing enzymes that help control sensitivity to various chemicals," says Hahn.

\section{The future looks wild}

Fish are far from the only wild model. Other researchers are examining how ground squirrels survive low body temperatures and hypoxic conditions during hibernation, and how those lessons could inform use of hypothermia during surgery or preservation of human organs for transplantation.

Naked mole rats can go 18 minutes without breathing, in part by switching metabolism from glucose to fructose and circumventing lactate buildup that shuts down glucose metabolism, a talent that might help treat heart attacks and strokes ${ }^{8}$. Pythons gorging on massive meals jumpstart their pancreatic beta cells, an effect that researchers would like to mimic to treat diabetes.

\section{"We can study traits at extreme ends of a spectrum, and gain a deeper understanding of the fundamental principles of life," says Misty Riddle.}

All of this focus on evolutionary adaptations in wild models may seem new, but it isn't. In 1929, Danish physiologist and Nobel laureate August Krogh declared that just about any physiological problem can be tackled by finding a wild organism that displays a similar trait ${ }^{9}$.

In that essay, Krogh discussed a tortoise with a unique trachea that provided an excellent model for the study of respiration, almost as if the animal had been created for that purpose. "I have no doubt that there is quite a number of animals which are similarly 'created' for special physiological purposes, but I am afraid that most of them are unknown to the men for whom they were 'created,' and we must apply to the zoologists to find them and lay our hands on them," he wrote.

Indeed, Krogh may have foreseen the long-term evolution of his field, if Riddle is correct. "We've learned a lot from focusing on a few 'models', but evolution has undoubtedly come up with solutions to life's problems that we cannot fathom. We can study traits at extreme ends of a spectrum, and gain a deeper understanding of the fundamental principles of life," she says.

\section{Jim Kling \\ Freelance writer, Bellingham, Washington, USA. e-mail: jkling@gmail.com}

Published online: 22 July 2019

https://doi.org/10.1038/s41684-019-0353-3

\section{References}

1. Hernan, A. et al. Mol. Ecol. 27, 4397-4416 (2018)

2. Peters, M. J. et al. Biol. Open 7, bio035576 (2018).

3. Kim, B. et al. Nat. Ecol. Evol. 3, 469-478 (2019).

4. McGaugh, S. E. et al. Nat. Commun. 5, 5307 (2014).

5. Reid et al. Genome Biol. Evol. 9, 659-676 (2017),

6. Kling, J. Lab. Anim. (NY) 47, 152 (2018).

7. Clark, B. W., Matson, C. W., Jung, D. \& Di Giulio, R. T. Aqut. Toxicol. 99, 232-240 (2010).

8. Park, T. J. et al. Science 21, 307-311 (2017).

9. Krogh, A. American Journal of Physiology 90 (1929) 